\title{
Water bucket colour preferences in horses
}

\author{
Fatih Yildirim ${ }^{a^{*}, \text { Ahmet Yildiz }}$
}

\begin{abstract}
There is a general agreement that horses have dichromatic colour vision with similar capabilities to those of human beings with red-green colour deficiencies. However, whether colour perception has an impact on equine preference for drinking water and how pronounced the colour stimulus might be for horses is still unknown. This study aimed to investigate the preference of horses for different coloured buckets (red, light green, yellow, green, light blue, turquoise) with different tones, in terms of lightness and darkness, measured by the amount of water consumed from each of them in an outdoor arena. Three Thoroughbred and 3 Haflinger horses were used in the study, and data were collected between 09:00 - 16:00 $\mathrm{h}$. The results showed that the average water consumption during this period can reach up to $25.74 \mathrm{~L}$ (at an average environmental temperature of $27.35^{\circ} \mathrm{C}$ ), and significantly more water had been consumed from the turquoise-coloured bucket than from the other colours. It was also observed that the light toned colours (turquoise, light blue) were preferred to dark ones (green, yellow, red). It is suggested to use water buckets that are turquoise and light blue. Further studies are needed to better understand the relationship between water consumption and colour vision.

Key words: bucket, colour, equine, preference assessment, water consumption.
\end{abstract}

\section{INTRODUCTION}

Most mammals have two cone pigments, one maximally sensitive to wavelengths near the middle of the spectrum (green/yellow) and one maximally sensitive to wavelengths at the short end of the visible spectrum (blue). Trichromatic colour vision is rare among mammals, occurring only in some primates (Rowe 2002). The wavelength-discrimination functions of normal trichromatic humans typically feature two spectral regions where discrimination is most acute (at $\sim 500 \mathrm{~nm}$ and $580 \mathrm{~nm}$ ), whereas analogous functions obtained from dichromats have a single region of acute discrimination $(\sim 500 \mathrm{~nm})$. Although dichromatic colour vision is common among mammals, it is not universal. This is because some mammalian species have retained only a single functional cone pigment and because numerous mammals are nocturnal, often having retinas that contain two types of cones, but with those two being only sparsely represented in the retina (Jacobs 2018).

Studies on the visual cones of horses have established that they have two cone pigments (Sandmann et al 1996, Carrol et al 2001, Hall et al 2006). Carroll et al (2001) reported that horses have dichromatic colour vision with two cone types that are sensitive to short and medium wavelengths, $428 \mathrm{~nm}$ peak and $539 \mathrm{~nm}$ peak, respectively. This means that horses have reduced colour vision compared to humans and, therefore, cannot distinguish between many of the colours (Spaas et al 2014).

Received: 06.01.2020.

Accepted: 24.04.2020.

a Department of Animal Science, Faculty of Veterinary Medicine, University of Atatürk, Erzurum, Turkey

*Corresponding author: F Yildirim; fatihyildirim@atauni.edu.tr
Previous behavioural studies using different methods indicate that horses are at least dichromatic with colour vision capabilities that are similar to those of human beings with red-green colour deficiency (Spaas et al 2014). Smith and Goldman (1999) stated that horses can discriminate the colours red, yellow, green and blue from various shades of grey, and thus have colour vision that is sensitive to short, medium and long wavelengths. However, a later study by Roth et al (2007) suggested that if dichromats do not perceive grey as qualitatively different from red, blue, green or yellow, an improved colour vision test could result from testing colours that are different from the neutral point instead of the classic grey versus colour tests. Blackmore et al (2008) suggested that horses can discriminate between yellow and blue, but that they may have deficiencies in discriminating red and green. However, there is still a lack of research on whether horses have a preference for certain colours.

Horses should drink water at every opportunity during training and competition, otherwise, they can experience a substantial loss of body weight, as well as dehydration (Muñoz et al 2017). Water is essential for gastrointestinal health, digestive function and body fluid balance, therefore, it is very important to ensure that horses drink enough water. Daily drinking water requirements range from 21 to $29 \mathrm{~L}$ per day for a maintenance $500 \mathrm{~kg}$ horse on a hay-based diet. However, this varies based on many conditions, including ambient air temperature and workload (NRC 2007). In this study, water consumption was used to determine the preference of bucket colour by horses. There is no uniformly accepted practice regarding the location of water buckets and horses are commonly provided water buckets while stabled (Akam et al 2017).

This study aimed to investigate the preference of horses for different coloured buckets (red, light green, yellow, green, light blue, turquoise) with different tones, in terms of lightness and darkness, measured by the amount of water consumed from each of them. 


\section{MATERIAL AND METHODS}

No permits or ethical committee approvals were required since the horses were not trained on any exercise during the assessment. To carry out the study, institutional approval was obtained in writing from the institution that owns the horses.

HORSES

Three Thoroughbred horses ( 1 mare, 2 geldings) and 3 Haflinger horses (3 mares) with a mean age of 10 years (range 6-18 years) (table 1) were observed. Their average body weight was calculated as $493.71 \pm 20 \mathrm{~kg}$ for the Haflingers and $476.47 \pm 20 \mathrm{~kg}$ for the Thoroughbred horses. The horses were not subjected to exercise throughout the study and their daily diet was provided in the same way, no additional food was given. A daily diet of $1-2 \mathrm{~kg}$ of roughage and $0.5-1 \mathrm{~kg}$ of concentrated feed per $100 \mathrm{~kg}$ body weight was provided and the horses were allowed to graze in the paddock. Equestrians and observers of the horses assessed whether the horses were healthy and in good condition. To examine the physiological status of horses used in the study, it was checked that mares were not pregnant or in the lactation period throughout the study. One horse of each breed was placed into two different paddock areas for 6 days during the study.

\section{BUCKETS AND COLOURS}

A total of 12 buckets of 6 different colours ( 2 buckets of each colour) were used in the study. The colours were red, light green, yellow, green, light blue, and turquoise. The buckets were all the same size $(35 \mathrm{~cm}$ diameter and $40 \mathrm{~cm}$ height), made of 0.5 -mm-thick galvanized sheet metal, and painted with the colours to be used in the study (figure 1).

The average colour for each image cluster is shown in table 2. The colour names given are those closest to the colours of the buckets and were obtained from the Image Color Summarizer 0.76@ software ${ }^{1}$. The RGB (red, green, blue), HSV (hue, saturation, value), LCH (lightness, chroma, hue angle) and Lab (lightness, red/green value, blue/yellow value) values were measured for all colours.

To determine the colours to be used in the study, a search was done on the colours that horses can and cannot see (Smith and Goldman 1999, Rowe 2002, Roth et al 2007, Blackmore et al 2008, Spaas et al 2014). Then, the colour mixtures were planned. Also, people with red colour blind disease were asked about the colours used in this study. Previously in the stud farm, only large buckets of grey colour were used to provide drinking water to the horses.

\section{WATER CONSUMED}

To calculate the amount of water drank by the horses from the buckets, the maximum amount of water consumed by horses daily (about $45 \mathrm{~L}$ ) was first determined. Considering the 7 hour period planned for the research (see below), it was decided that $15 \mathrm{~L}$ of water daily would be provided per bucket. To avoid errors when calculating the litres in

1 Krzywinski M. 2019. Image Color Summarizer 0.76 @; http:// mkweb.bcgsc.ca/color-summarizer/?analyze

Table 1. The horses used in the study.

\begin{tabular}{llccc}
\hline Horses & \multicolumn{1}{c}{ Breed } & $\begin{array}{c}\text { Neck circumference } \\
(\mathrm{cm})\end{array}$ & $\begin{array}{c}\text { Body length } \\
(\mathrm{cm})\end{array}$ & $\begin{array}{c}\text { Chest circumference } \\
(\mathrm{cm})\end{array}$ \\
\hline H1 & Haflinger & 107 & 166 & 191 \\
H2 & Thoroughbred & 78 & 157 & 183 \\
H3 & Haflinger & 110 & 174 & 187 \\
H4 & Thoroughbred & 80 & 174 & 189 \\
H5 & Haflinger & 94 & 161 & 184 \\
H6 & Thoroughbred & 96 & 168 & 181 \\
\hline
\end{tabular}

Table 2. Paints used to colour the buckets.

\begin{tabular}{llcccc}
\hline Colors & \multicolumn{1}{c}{ Name $^{1}$} & RGB & HSV & LCH & Lab \\
\hline Red & Sangria & 1451010 & 3609357 & 306437 & 305139 \\
Yellow & Dark goldenrod & 2231608 & 429688 & 707479 & 701373 \\
Green & English holly & 195223 & 1256321 & 1925142 & $19-2015$ \\
Light green & Cobalt green & 5013860 & 1266354 & 5155141 & $51-4334$ \\
Light blue & Hemisphere & 63147193 & 2016775 & 5833251 & $58-11-31$ \\
Turquoise & Java & 57156158 & 1816462 & 5929199 & $59-28-9$ \\
\hline
\end{tabular}




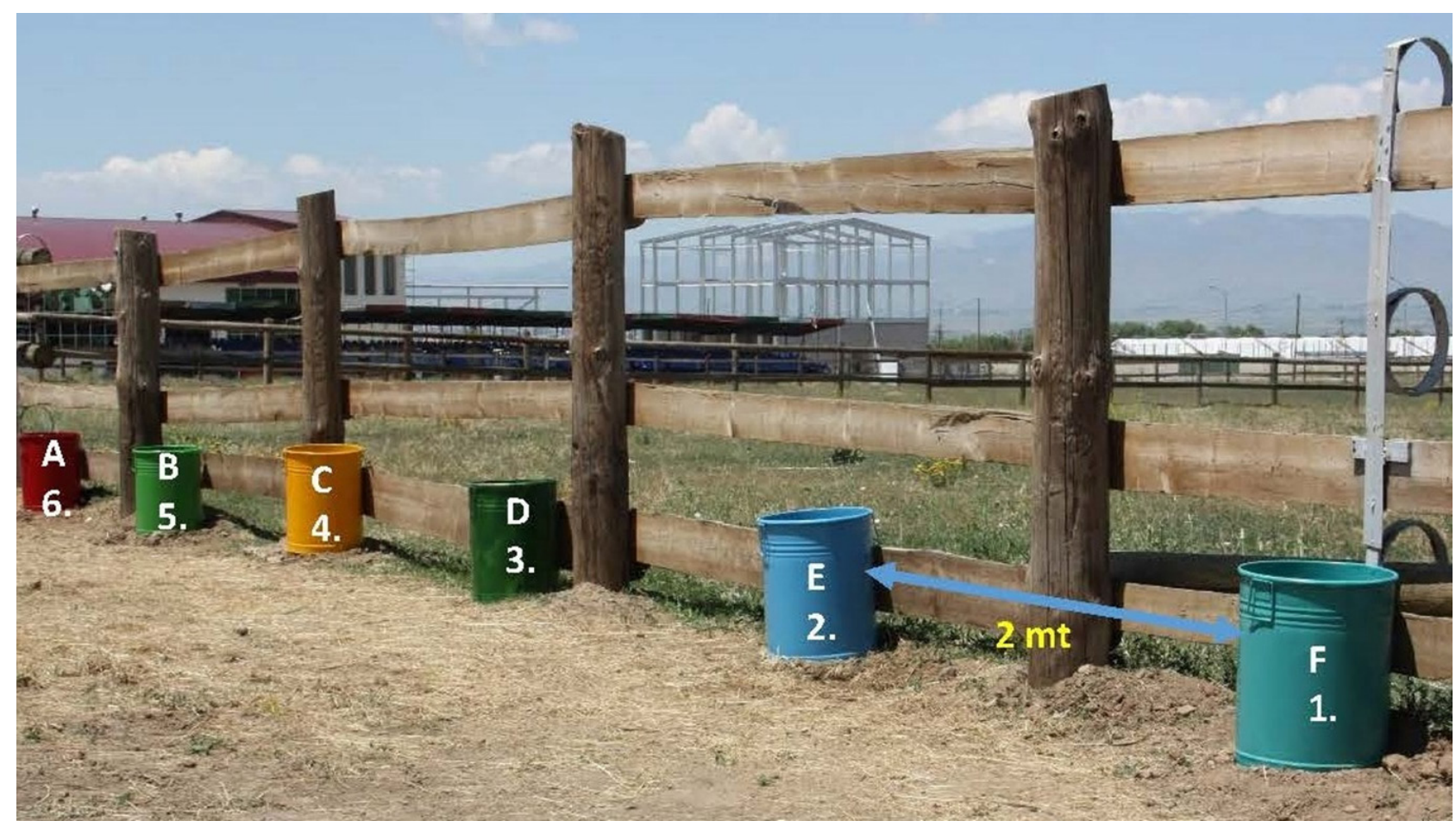

Figure 1. Colours and rows of the buckets used in the study. (A) red, (B) light green, (C) yellow (D) green (E) light blue, (F) turquoise. (1.) 1 st row, (2.) 2nd row, (3.) 3rd row, (4.) 4th row, (5.) 5th row, (6.) 6th row. A total of 12 buckets of 6 different colors (2 buckets of each color) were used in the study. There were about two meters between the buckets, which were attached to the side fences with rope. Each day, the buckets were shifted by one row, and thus the buckets were each tested in all positions.

the study area, a hard (nonpliant) and narrow-mouthed (preventing water from spilling into the environment) plastic jerrycan was used to pour $15 \mathrm{~L}$ of water into the coloured buckets. The amount of water poured into this plastic jerrycan was weighed as gram with a weighing instrument (TEM Industry and Trade Limited Company®) for easy measurement.

\section{EXPERIMENTAL DESIGN}

The study was carried out between 09:00 and 16:00 $\mathrm{h}$ in two outdoor paddock areas surrounded by fences, and the paddocks had no protection from the sun or other weather inclement. Each paddock had an area of about $20 \times 40 \mathrm{~m}^{2}$ (figure 2). The evaluation of horses from two different breeds placed side by side in the paddock areas provided equal environmental factors, making the rate of exposure to environmental factors similar. The animals stayed $7 \mathrm{~h}$ (09:00-16:00) per day in the paddock and then went back to their stalls. The observation of one horse of each breed lasted 6 consecutive days to allow the horse to see all possible rows of buckets.

During the 18-day study period, each colour bucket was tested 18 times on each breed (3 horses x 6 rows), or 36 times in a total of two breeds. Six buckets, one in each of the six colours, were placed in each paddock along with one horse, either a Thoroughbred or Haflinger. The buckets were attached to the side fences with a rope and there was a distance of two meters between them. The buckets were shifted by one row everyday, to test each one in every single position before the next horse group (one Thoroughbred and one Haflinger) came along. In this way, the effect of horse preference for bucket location was minimised.

The water $(15 \mathrm{~L})$ in the plastic jerrycan was poured into the coloured buckets at 09:00 and later the remaining water contents in the buckets were weighed again (grams) at 16:00, to calculate water consumption. The amount of water consumed was determined by removing it from the total water put into the buckets. The data ultimately obtained from this weighing were converted to litres. If the drinking water in the bucket was depleted during the research period, more water was provided and included in the calculation. Water consumption restrictions were not applied to animals before and after the study hours. There was always water in front of the horses leaving the study area and being taken to their stalls. The average temperature during the research period (July) was 27.35 ${ }^{\circ} \mathrm{C}$ and relative humidity was $24.0 \%$.

\section{STATISTICAL ANALYSIS}

The results of this study are presented as means and standard error of the mean (SE). Differences were considered 


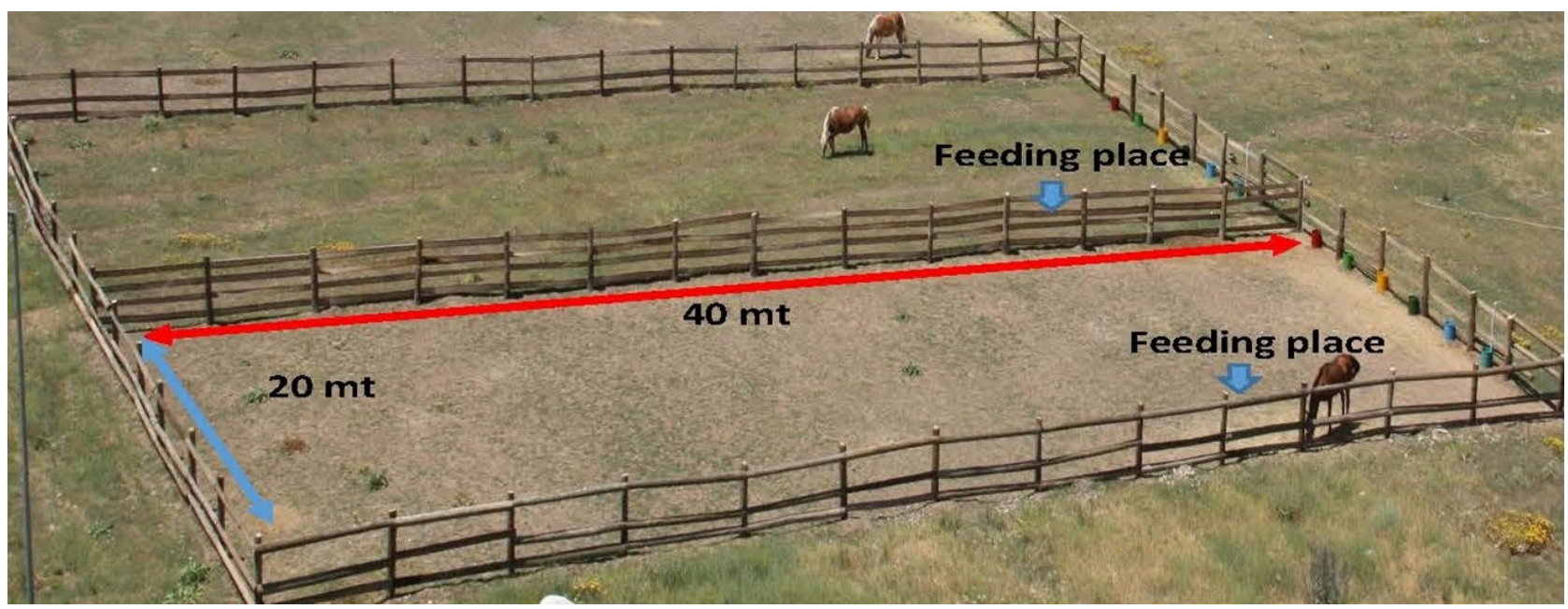

Figure 2. The paddock areas surrounded by fences used in the study. The paddocks had no protection for sun or other weather inclement. Each paddock had an area of about $20 \times 40 \mathrm{~m}^{2}$. There were two different breeds (Thoroughbred and Haflinger) in each paddock.

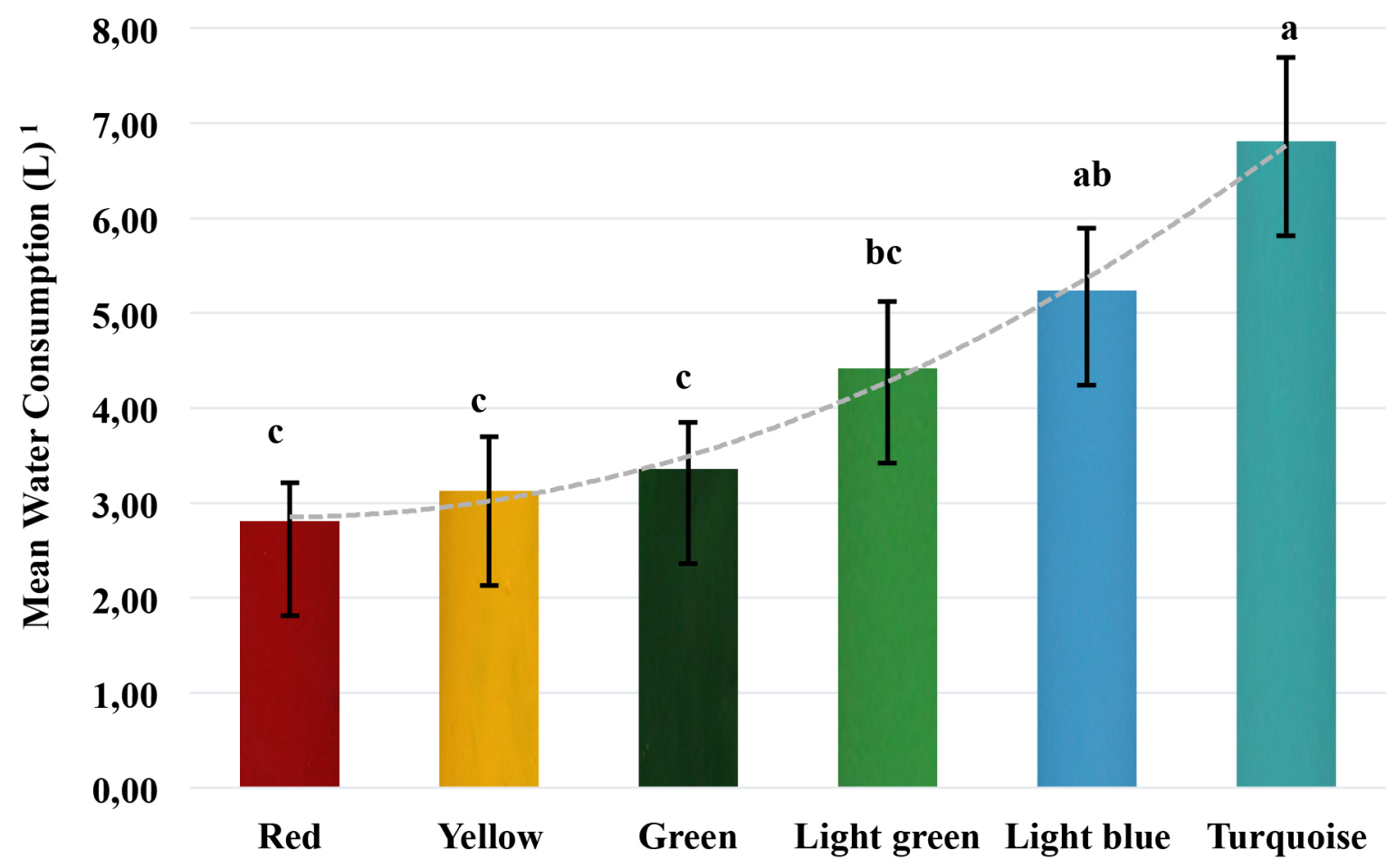

\section{Bucket Colours}

Figure 3. Mean (columns) and their standard errors (bars) for water amount consumed from coloured buckets by horses. a, b, c: Values with different superscripts in the same column for each section are significantly different $(P<0.05)$. ( 2 horses, 18 days $=36$ observations for each colour).

${ }^{1}$ Mean water consumption values cover 7 hours a day for each horse.

significant at $P<0.05$. In the case of an analysis (general linear model) revealing a significant difference, the differences among specific groups were then analysed using Duncan's multiple comparison post-hoc test $(P<0.05)$. Data were analysed by using IBM SPSS Statistics for Windows (Version 20, IBM Corp., Armonk, NY, USA).

\section{RESULTS}

The average ( 7 hours daily) water consumption of each horse was calculated as $4.29 \mathrm{~L}$, totalling $25.74 \mathrm{~L}$ from 6 buckets. Figure 3 shows the amount of water consumed by the horses during the complete trial. The results showed 
significant differences between bucket colours $(P<0.001)$. The horses preferred to drink water from the turquoise buckets $(6.81 \pm 0.88 \mathrm{~L})$ while the red buckets $(2.81 \pm$ $0.40 \mathrm{~L}$ ) had the lowest water consumption. The preferences for the remaining colours, from highest to lowest, were as follows: light blue $(5.24 \pm 0.65 \mathrm{~L})$, light green $(4.42 \pm 0.70 \mathrm{~L})$, green $(3.36 \pm 0.49 \mathrm{~L})$ and yellow $(3.13$ $\pm 0.57 \mathrm{~L}$ ). Also, the upper and lower bounds of the water consumption according to $95 \%$ confidence interval were calculated as $1.56 \mathrm{~L}$ (red colour) and $8.06 \mathrm{~L}$ (turquoise colour), respectively.

The assessment of the lightness and darkness tones of the colours used in this research (figure 3) showed a preference of horses for the light blue bucket after the turquoise bucket, indicating that horses are more inclined toward different tones of blue colours (turquoise, light blue). Besides, when the results were examined in terms of light colour tones, it was observed that the most preferred turquoise and light blue rather than dark tone colours (red, yellow and green).

\section{DISCUSSION}

Daily water consumption of horses range from 21 to $29 \mathrm{~L}$ (NRC 2007), while other report 30.2 to $45.4 \mathrm{~L}$ fresh and drinking water per day under normal environmental conditions (Evans 2000). Pagan et al (2017) reported an increased water intake under conditions of increasing daily temperature that ranges from $21^{\circ} \mathrm{C}$ to $28^{\circ} \mathrm{C}$. In this study, the average 7 hours water consumption of a horse was $25.74 \mathrm{~L}$. Water consumption could vary based on many factors (environmental temperature, humidity, feed, physiological status, exercise, etc). However, those factors were minimised in the present study to assess the horse preference for a colour, instead of daily water consumption.

There are studies showing peaks in spectral sensitivity, meaning that horses see blue and green colours better than other colours (Macuda and Timney 1999, Geisbauer et al 2004). Other researchers have stated that horses can discriminate yellow and blue, but that they may have deficiencies in discriminating red and green (Blackmore et al 2008). Also, Hanggi et al (2007) suggest that horses are dichromats with colour vision capabilities similar to those of humans with red-green colour deficiencies, but can perceive blue. Horses have two cone pigments in retinas, and each of these cones has pigments that can separate green/yellow and blue. They can generally distinguish some colours from others if green/yellow and blue main colours are used. In this study, both blue and yellow colours were evaluated, and the mixture of them. The difference with the other colours used in this research was also evaluated using the red colour, which horses do not see clearly.

In general, most of the studies (Spaas et al 2014, Smith and Goldman 1999, Roth et al 2007, Blackmore et al 2008) on horse vision have been conducted with certain colour tones (e.g., red, yellow, blue and green), and have not sufficiently examined variations of those particular tones. In this study, different tones (e.g. light green, light blue, and turquoise) that might affect the vision of the horse were used, and different results were obtained. Results shown in figure 3 indicate that horses preferred to drink water from the turquoise colour bucket, which is a mixture of blue and green. Also, the animals were more attracted to the light colour tones (light blue and light green) than to the dark colour tones (red and green). However, Davis et al (2017) reported that stalled horses had no preference when given the option of drinking out of light (yellow) or dark (black) buckets. In this study, a statistically significant difference was found between the amount of water consumed from coloured buckets $(P<0.001)$, but there was no difference in the study of Davis et al (2017) $(P>0.05)$.

Hall and Cassaday (2006) reported that after an initial presentation of colours on the ground, yellow, white, black and blue colours were found to cause a greater number of adverse reactions compared to green, red, brown and grey colours, however, we observed that the light blue and turquoise bucket colours used in the present study were preferred over red and yellow bucket colours.

It can be concluded that horses significantly preferred consuming water from the turquoise-coloured buckets when compared to other colours such as light green, green, yellow, and red. Besides, the water consumption from light tone coloured buckets (turquoise, light blue) was higher than in dark tone ones (green, yellow, red). Therefore, it is suggested to use turquoise, or light blue water buckets. Further investigations on the relationship between the water consumption, colour and tone of buckets are needed to help better understand the correlation between colour vision, preference, and water consumption.

\section{ACKNOWLEDGEMENTS}

The authors would like to thank Murat TEMEL, Ruhsane KALAYCI, Ömer GÜLSU, Batuhan NARMANLI, Alihan TURHAN, and Atatürk University Horse Breeding and Training staff and volunteers for providing assistance and subjects for use in this study.

\section{REFERENCES}

Akam BC, Siehr BA, Davis AT, FitzSimmons JA. 2017. Effect of bucket placement on water intake. J Equine Vet Sci 52, 107.

Blackmore TL, Foster TM, Sumpter CE, Temple W. 2008. An investigation of colour discrimination with horses (Equus caballus). Behav Process 78, 387-396.

Carroll J, Murphy CJ, Neitz M, Hoeve JN, Neitz J. 2001. Photopigment basis for dichromatic color vision in the horse. $J$ Vis 1, 80-87.

Davis AT, Siehr BA, Akam BC, FitzSimmons JA. 2017. Effect of bucket color on water intake in horses. J Equine Vet Sci 52, 106.

Evans JW. 2000. Horses: A guide yo selection, care, and enjoyment. $3^{\text {th }}$ ed. Henry Holt and Company, New York, USA.

Geisbauer G, Griebel U, Schmid A, Timney B. 2004. Brightness discrimination and neutral point testing in the horse. Can J Zool 82, 660-670.

Hall CA, Cassaday HJ, Vincent CJ, Derrington AM. 2006. Cone excitation ratios correlate with color discrimination performance in the horse (Equus caballus). J Comp Psychol 120, 438-448. 
Hall CA, Cassaday HJ. 2006. An investigation into the effect of floor colour on the behaviour of the horse. Appl Anim Behav Sci 99, 301-314.

Hanggi EB, Ingersoll JF, Waggoner TL. 2007. Color vision in horses (Equus caballus): Deficiencies identified using a pseudoisochromatic plate test. J Comp Psychol 121, 65.

Jacobs GH. 2018. Photopigments and the dimensionality of animal color vision. Neurosci Biobehav Rev 86, 108-130.

Macuda T, Timney B. 1999. Luminance and chromatic discrimination in the horse (Equus caballus). Behav Process 44, 301-307.

Muñoz A, Castejón-Riber C, Riber C, Esgueva M, Trigo P, et al. 2017. Current knowledge of pathologic mechanisms and derived practical applications to prevent metabolic disturbances and exhaustion in the endurance horse. J Equine Vet Sci 51, 24-33.

NRC, National Research Council. 2007. Nutrient requirements of horses. $6^{\text {th }}$ ed. The National Academies Press. Washington, DC, USA.
Pagan JD, Hauss A, Swanhall A, Ford E, Rugg E, et al. 2017. Water, concentrate, and hay intake in thoroughbred racehorses. $J$ Equine Vet Sci 52, 89.

Roth LSV, Balkenius A, Kelber A. 2007. Colour perception in a dichromat. J Exp Biol 210, 2795-2800.

Rowe MH. 2002. Trichromatic Color Vision in Primates. News Physiol Sci 17, 93-98.

Sandmann D, Boycott BB, Peichl L. 1996. Blue-cone horizontal cells in the retinae of horses and other Equidae. J Neurosci 16, 3381-3396.

Smith S, Goldman L. 1999. Color discrimination in horses. Appl Anim Behav Sci 62, 13-25.

Spaas J, Helsen WF, Adriaenssens M, Broeckx S, Duchateau L, et al. 2014. Correlation between dichromatic colour vision and jumping performance in horses. Vet $J$ 202, 166-171. 